\title{
Respuesta a la carta al editor respecto del artículo: "Negligencia: modalidad subestimada del maltrato al infante"
}

\author{
Reply to the letter to the editor in relation to the article: \\ Neglect: underestimated from of child abuse
}

\section{Estimado Dr. Francisco Espinosa Rosales}

(Editor en Jefe de Acta Pediátrica de México)

En respuesta a la carta al Editor de Acta Pediatrica de México, que ha enviado el Dr. Óscar Gabriel Carballido Moreno y por estar fuera del país la Dra. Mónica Rodríguez González, me permitió responder a la misma, con las siguientes consideraciones.

Es muy interesante y halagador que un médico en el Servicio Social haya leído el artículo: "Negligencia: modalidad subestimada del maltrato al infante" aparecido en el Volúmen 40 Número 2 del año 2019 de Acta Pediatrica de México y captado la importancia que nosotros, como autores, creemos se le debe dar a esta modalidad de maltrato infantil, en la clínica pediátrica diaria. Pero lo más interesante, es que el Dr. Carballido Moreno tomó la iniciativa de proponer una definición puntual, que modifica y enriquece sustancialmente numerosas definiciones internacionales y nacionales. ${ }^{1}$

El objetivo de esta propuesta es auxiliar a los clínicos para sospechar o establecer el diagnóstico de probable negligencia, una de las 4 modalidades de maltrato infantil. Por supuesto, la sugerencia que plantea el Dr. Carballido Moreno es magnífica porque considera tres aspectos básicos inmersos en dicha definición y estos son:

1. El primer elemento a considerar es la existencia de una "intencionalidad" o "daño no accidental", como se describe en la literatura anglosajona, que causa el agravio físico o emocional en una niña, niño o adolescente. Esta condición establece la gran diferencia entre la violencia contra una niña o niño y el maltrato infantil. ${ }^{2-3}$

Coordinador de la CEAMI, Instituto Nacional de Pediatría, Ciudad de México.

Correspondencia

Arturo Loredo Abdalá

cainm_inp@hotmail.com

Este artículo debe citarse como

Loredo Abdalá A. Respuesta a la carta al editor respecto del artículo: "Negligencia: modalidad subestimada del maltrato al infante". Acta Pediatr Mex. 2109;40(5):301-02. 
2. En segundo término, está el no atender, en el análisis inicial de estos casos, la condición socioeconómica de los padres o cuidadores, ya que esta situación puede generar una desviación al analizar la causalidad del problema. Con frecuencia, la existencia de un nivel socioeconómico bajo puede desorientar al clínico cuando lo considera como el factor causante del problema, situación que no necesariamente es verdad. ${ }^{4-6}$

3. Finalmente, el que, en diversas definiciones de organismos internacionales, no consideran la repercusión física y emocional que la víctima desarrolla a corto, mediano o largo plazo como consecuencia de la agresión sufrida. Esta situación es una gran limitante para el entendimiento global del problema. ${ }^{7-9}$

La propuesta del Dr. Óscar Gabriel Carballido Moreno muy probablemente está basada en la definición desarrollada en la Clínica de Atención Integral al Niño Maltratado del Instituto Nacional de Pediatría (CAINM-INP) y publicada en el año 2001. Los resultados obtenidos con su empleo por más de 10 años nos permitieron, en su momento, incluirla en diversos artículos. ${ }^{10-12}$

Ojalá y la mayoría de los lectores de Acta Pediátrica de México no sólo tengan el anhelo e impulso de leer los artículos publicados en nuestra revista, sino también proponer comentarios positivos y enriquecedores sobre los mismos, como lo ha hecho el Dr. Óscar Gabriel Carballido Moreno. Es muy deseable que este ejemplo prolifere, principalmente en los médicos jóvenes.

\section{REFERENCIAS}

1. Rodríguez-González M, Loredo-Abdalá A. Negligencia: modalidad subestimada del maltrato al infante. Acta Pediatr Mex. 2019;40(2):85-92

2. Reading R, Bissell S, Goldhagen J, Harwin J, Masson S, Parton $\mathrm{N}$, et al. Promotion of children's and prevention of child maltreatment. Lancet 2009; 373: 332-43. DOI:10.1016/ S0140-6736(08)61709-2

3. CENETEC, GPC. Detección Temprana del Abuso Físico desde el Nacimiento hasta los 12 años. DIF-400-09.

4. Polansky NA, De Saix C y Sharlin SA. (1972). Child neglect. Understanding and reaching the parent. Washington: Child Welfare League of America.

5. Del Águila -Escobedo A. Violencia y estrés infantil: ¿̇está en juego nuestro futuro? Acta Médica Peruana. 2015; 32: $71-83$

6. CNDH México. Erradicar la violencia contra niñas, niños y adolescentes. 2018

7. De Panfilis. Child Neglect: A Guide for Prevention, Assessment, and Intervention. U.S. Department of Health and Human Services. 2006

8. Organización Panamericana de la Salud, Organización Mundial de la Salud. Informe mundial sobre la violencia y la salud. 2002

9. Modovar C, Ubeda M. La violencia en la primera infancia. UNICEF. Ciudad de Panamá. 2017

10. Perea- Martínez A, Loredo-Abdalá A, Trejo-Hernández J, Baez- Medina $V$ y cols. El maltrato al menor: propuesta de una definición integral. Bol Med Hosp Infant Mex 2001; 58:251-8

11. Loredo Abdalá A, Monroy Llaguno D, Casas Muñoz A. El maltrato infantil: Conceptos básicos de una patología médico social legal. Revista de la Facultad de Medicina (México); 2013; 56: 5-23.

12. Loredo-Abdalá A. Maltrato infantil: consideraciones básicas para el diagnóstico de las formas más preponderantes. Acta Pediatr Mex. 2008; 29:255-61.

Atentamente

\section{Dr. Arturo Loredo Abdalá}

